\title{
Effect of platelet-rich plasma on bone engineering with an alloplastic substitute containing BMP2
}

\author{
Kaori Yoshida ${ }^{a}$, Yoshinori Sumita ${ }^{\mathrm{b}, *}$, Eriko Marukawa ${ }^{\mathrm{c}}$, Masaru Harashima $^{\mathrm{d}}$ and \\ Izumi Asahina ${ }^{\mathrm{b}}$ \\ ${ }^{a}$ Department of Oral Surgery, Kamagaya General Hospital, Chiba, Japan \\ ${ }^{\mathrm{b}}$ Department of Regenerative Oral Surgery, Nagasaki University Graduate School of Biomedical \\ Sciences, Nagasaki, Japan \\ ${ }^{\mathrm{c}}$ Oral and Maxillofacial Surgery, Department of Oral Restitution, Division of Oral Health Sciences, \\ Tokyo Medical and Dental University Graduate School, Tokyo, Japan \\ ${ }^{\mathrm{d}}$ Harashima Dental Clinic, Tokyo, Japan
}

Received 11 January 2012

Accepted 27 August 2012

\begin{abstract}
A robust method for inducing bone-formation without an autograft has not been established. Currently, both plateletrich plasma (PRP) and bone morphogenetic protein (BMP) have been widely investigated for their clinical use in such cases. However, their synergistic effect is still controversial and previously shown diversity of this effect depends on various factors such as the bone substitutes involved. In this study, we investigated the synergistic effect of PRP and BMP2 on an alloplastic substitute as potentiators to induce in vivo bone-formation. A $10 \mathrm{~mm}$ diameter bony defect in rabbit calvarium was reconstructed using biphasic calcium phosphate (BCP) ceramics with or without PRP, recombinant human (rh) BMP2, and their combination. At 6 and 12 weeks after implantation, rabbits were euthanized and the radiographic and histomorphometric features of bone reconstruction were analyzed. The results showed that defects filled by rhBMP2/BCP with or without PRP had high bone density at 6 and 12 weeks in radiological evaluation. However, in histomorphometric analysis, the defects filled by rhBMP2/BCP with PRP showed significant new bone formation compared with that by rhBMP2/BCP without PRP, especially at 6 weeks. We propose that the synergistic effect of PRP and rhBMP2 gives highly osteoinductive properties to alloplastic substitutes in vivo.

Keywords: Bone regeneration, bone morphogenetic protein, platelet-rich plasma, alloplastic bone substitute
\end{abstract}

\section{Introduction}

Bone defects due to disease or trauma that do not heal spontaneously require bone reconstruction to restore bone function. Autogenous bone grafts, considered to be the gold standard, are especially used to regenerate bony defects in the craniofacial region [1-6]. However, allografts, xenografts, or alloplastic bone substitutes are currently employed in clinics because autografts involve donor site morbidity and may lack osteogenic potential [5,7-9]. In particular, alloplastic bone substitutes, such as hydroxyapatite

\footnotetext{
*Address for correspondence: Associate Professor Yoshinori Sumita, 1-7-1 Sakamoto, Nagasaki 852-8588, Japan. Tel.: +81 95819 7701; Fax: +81 95819 7705; E-mail: y-sumita@ nagasaki-u.ac.jp.
} 
(HA) or tricalcium-phosphate (TCP) ceramics, are very promising to avoid the disadvantage of autografts because their geometry and degradation properties can be adapted to the local demands, while infection transmission can be avoided [6,7]. Such alloplastic substitutes have excellent biocompatible and osteoconductive abilities, but they lack osteoinductive properties $[10,11]$. Therefore, growth factors and morphogens have received increasing attention as materials that can confer osteoinducibility to alloplastic bone substitutes.

One of the bone morphogenetic proteins (BMPs), recombinant human (rh) BMP2, has been shown to induce bone formation in a variety of indications [10,12-16]. A large number of animal and human studies have demonstrated the successful use of rhBMP2 to induce bone regeneration for large bony defects such as mandibular resection and cleft palate defects [14,17-19]. For such trials, various biomaterials have been examined as carriers for rhBMP2 and rhBMP2 has been shown to significantly enhance bone regeneration in rabbit calvaria when delivered by an alloplastic substitute containing HA/TCP [14]. However, direct implantation of high doses of rhBMP2 is known to induce substantial swelling that may cause the obstruction of the airway when applied to oral and cervical areas [20,21]. An efficient delivery method for the clinical use of rhBMP2 remains to be developed.

On the other hand, platelet-rich plasma (PRP) is clinically used as an autologous blood product to stimulate tissue regeneration in various bony defects and in guided bone regeneration around dental implants $[8,22]$. The capacity of PRP to stimulate bone regeneration is believed to be due to the stimulatory activities of the growth factors released from activated platelets on progenitor cells and vascularization at local sites [22-24]. Platelets may contain novel potentiators of BMPs that stimulate osteoblastic differentiation of progenitor cells in vitro [22].

The aim of this study is to test whether PRP in combination with biphasic calcium phosphate (BCP) ceramics containing rhBMP2 enhances bone formation. We hypothesized that the addition of osteoinducibility to the alloplastic substitutes by the synergistic effect of PRP and rhBMP2 could provide a highly relevant approach to clinical bone reconstruction in the near future.

\section{Materials and methods}

All procedures with animals were carried out under protocols approved by the Facility Animal Care Committee of Tokyo Medical and Dental University.

\subsection{Preparation of platelet-rich plasma}

Two milliliters of citrate phosphate dextrose (CPD; Terumo Corporation, Tokyo, Japan) was aspirated in a $10 \mathrm{ml}$ plastic syringe as an anticoagulant, and then $10 \mathrm{ml}$ of venous blood was drawn from the otic vein of Japanese white rabbits (Sankyo Lab Service Corp., Tokyo, Japan) into the syringe several minutes before starting surgery. Blood mixed with CPD was transferred into a $15 \mathrm{ml}$ centrifuge tube. An aliquot was removed to determine the number of blood platelets. The number was counted using a cell counter (SE9000, Sysmex Corporation, Kobe, Japan). The blood was centrifuged at 1,500 $\times g$ for $10 \mathrm{~min}$ to separate the platelet-poor plasma (PPP) from the erythrocytes and the buffy coat, which contains the platelets and leukocytes. After removing the PPP fraction, the remnant was centrifuged again at $800 \times g$ for $8 \mathrm{~min}$ to allow further separation of the buffy coat from the red blood cells. This buffy coat/plasma fraction is the PRP used in the platelet gel. The number of blood platelets was counted with the cell counter. 


\subsection{Absorption of bone morphogenetic protein into biphasic calcium phosphate ceramics}

In this study, we used biphasic calcium phosphate (BCP) (provided by Sumitomo Osaka Cement Corp. Ltd., Funabashi, Japan) as a carrier of BMP as described previously. The ceramic is composed of $75 \%$ tricalcium phosphate (TCP) and $25 \%$ hydroxyapatite (HA). It was sintered at $900^{\circ} \mathrm{C}$. This process yielded microporous BCP particles approximately $200-500 \mu \mathrm{m}$ in diameter. The size of the micropores ranged from 0.5 to $2 \mu \mathrm{m}$. The rhBMP2 solution was prepared by dissolving $120 \mu \mathrm{g}$ of rhBMP2 (provided by Astellas Pharm Inc., Tokyo, Japan) in $1.2 \mathrm{ml}$ of $5 \mathrm{mM}$ sodium glutamate, $2.5 \%$ glycin, $0.5 \%$ sucrose, and $0.01 \%$ Tween 80 . The rhBMP2 solution $(10 \mu \mathrm{g} / 100 \mu \mathrm{l})$ was added to $100 \mathrm{mg}$ of BCP particles and then lyophilized overnight. Regarding the retention of rhBMP2 by BCP particles, we measured the concentration of rhBMP2 remaining in the supernatant by RH-HPLC at the absorbance of $214 \mathrm{~nm}$. The amount of rhBMP2 absorbed by BCP particles after 120 min revealed that the range of rhBMP2 adsorption was $91.5 \%$ [10]. This indicates that BCP particles work as good delivery system of rhBMP2.

\subsection{Platelet-rich plasma gel formation}

The PRP application requires initiating the coagulation process by the addition of calcium chloride and thrombin: 5,000 units of topical bovine thrombin (Sankyo Co. Ltd., Tokyo, Japan) were dissolved in $5 \mathrm{ml}$ of $10 \%$ calcium chloride (Otsuka Pharmaceutical Co. Ltd., Tokyo, Japan). Then, $0.3 \mathrm{ml}$ of PRP was poured into $100 \mathrm{mg}$ of BCP particles in a glass dappen dish and a few drops of calcium chloride/thrombin solution was added and mixed. The PRP coagulated immediately with the BCP particles (Fig. 1(a)).

\subsection{Surgical procedure}

Eighteen male Japanese white rabbits (Sankyo Lab Service Corp., Tokyo, Japan), each weighing $3 \mathrm{~kg}$, were used for this study. Two bony defects in the calvarium were created in each animal. The following five treatment modalities were randomly allocated to the 36 defects: (1) empty (Control): $n=6$ and 6 defects; (2) only BCP particles (BCP): $n=6$ and 6 defects; (3) BCP particles with PRP (PRP/BCP): $n=6$ and 12 defects; (4) rhBMP2-impregnated BCP particles (BMP2/BCP): $n=6$ and 6 defects; and (5) rhBMP2-impregnated BCP particles with PRP (PRP/BMP2/BCP): $n=6$ and 6 defects.

Rabbits were anesthetized with an intramuscular injection of ketamine hydrochloride $(1 \mathrm{mg} / \mathrm{kg})$. The surgical site was disinfected with iodine and $70 \%$ ethanol, and a linear incision was made in the sagittal

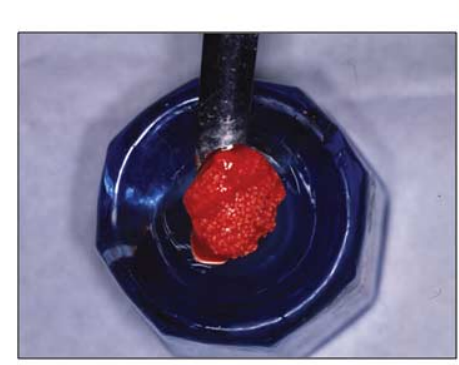

(a)

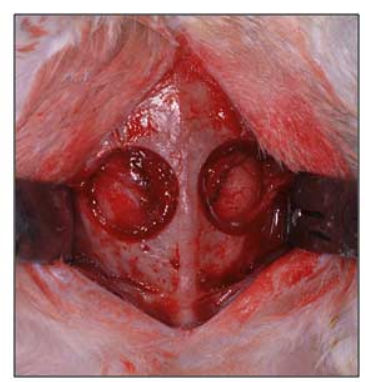

(b)

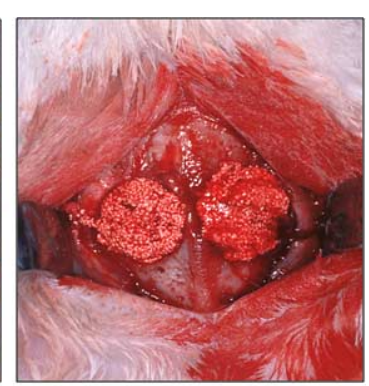

(c)

Fig. 1. (a) The dappen glass with PRP gel which was coagulated with BCP particles. (b) $10 \mathrm{~mm}$ in diameter of bone defects in bilateral calvaria. (c) Each graft was implanted into the defects. (Colors are visible in the online version of the article; http://dx.doi.org/10.3233/BME-130741.) 
plane across the cranium. A full-thickness flap including the periosteum was reflected and the parietal bone was exposed. Bone defects, $10 \mathrm{~mm}$ in diameter, on both halves of the calvarium were made using a saline-cooled trephine drill (Fig. 1(b)). Extreme care was taken to avoid injury to the brain. After removal of the trephined calvarial bone, individual grafts were implanted into the defects (Fig. 1(c)). No other carrier and no other regenerative materials were placed in the defects of the control group. The periosteum and skin were closed using 4-0 nylon sutures.

\subsection{Tissue harvest and radiological analysis}

Rabbits were killed at either 6 or 12 weeks following surgery. Each Calvarium including surrounding soft tissues was resected as a block and placed in $70 \%$ ethanol for fixation. Radiographs of the harvested specimens were taken using a DH158 HM X-ray unit (Hitachi, Japan) operating at $43 \mathrm{kV}$ and $100 \mathrm{~mA}$ with an exposure time of 3 min with ultrahigh contrast mammography film (X-OMATL Kodak, Rochester, NY). The images on the films were transferred to a computer and the density of each defect was calculated using National Institute of Health (NIH) image software, version 1.62 (National Institutes of Health, Bethesda, MD). Density calibration of NIH images ranged in value from 0 pixels (black) to 255 pixels (white).

\subsection{Histological analysis}

The specimens placed in 70\% ethanol were dehydrated with a graded series of ethanol and embedded in methylmethacrylate. Each block was sectioned to about 50- $\mu \mathrm{m}$ thickness using the cutting grinding system (EXACT, Hamburg, Germany) and the sections were stained with toluidine blue for light microscopy. Microscopic examination was performed on the stained sections. To access the bone formation, we examined the sections from the center of defects at the frontal plane of the calvarium (six sections from each of the six specimens per group). The images of the specimens were transferred to a computer and the area of new bone in each defect was calculated by NIH image software, version 1.62. The new bone area was expressed as pixels in the area.

\subsection{Statistical analysis}

All experimental values are presented as the mean \pm standard deviation. Means were analyzed using one-way analysis of variance. Dunnett's multiple comparison t-test was used to detect any significant differences within each group for the radiological and histological bone formation area. $P<0.05$ was considered significant.

\section{Results}

\subsection{The number of blood platelets}

The average number of blood platelets in the initial blood sample was $(35.1 \pm 0.95) \times 10^{4} / \mathrm{mm}^{3}$. The average number of blood platelets in the PRP sample was $(78.1 \pm 1.62) \times 10^{4} / \mathrm{mm}^{3}$. The preparation of PRP increased the blood platelet count approximately 2.23-fold compared with the initial concentration in whole blood. 


\subsection{Radiological evaluation}

The radiographs of the calvaria harvested at 12 weeks post-transplantation are shown in Fig. 2. Only a slight radiopaque image could be found at the defect site in the control group (Fig. 2(a)), while the radiographs of the BCP group showed scattered BCP particles at the periphery of the defect site (Fig. 2(a)). On the other hand, higher radiodensity with the remaining BCP particles was shown in the entire defect of PRP/BCP group (Fig. 2(b)). The fibrin component of PRP seemed to help the binding of BCP particles. Furthermore, the defects in both the BMP2/BCP and PRP/BMP2/BCP groups were filled with highdensity radiopaque tissues combined with the BCP particles (Fig. 2(c)). The edge of the high-density area in these defects became vague and these images seemed to suggest new bone formation.

Quantitative evaluation of the radiopaque areas using NIH imaging software is shown in Fig. 2(d). At 12 weeks post-transplantation, the radiopaque area in the defects of the PRP/BCP group increased compared with that of the BCP group. Furthermore, the defects in the PRP/BMP2/BCP group showed a higher radiopaque area compared with that in the BMP2/BCP group at both 6 and 12 weeks. However, there were no statistically significant differences among these groups.

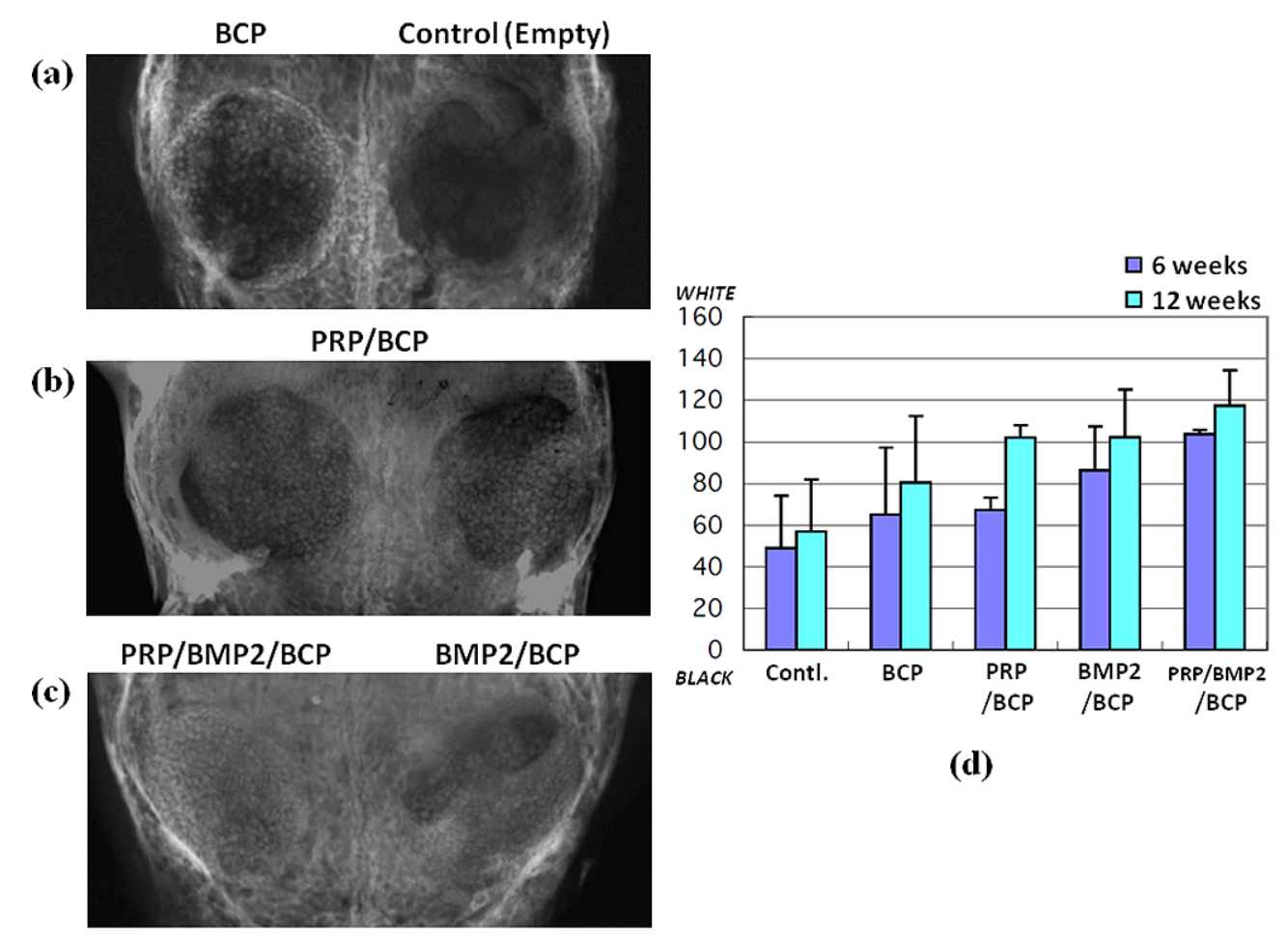

Fig. 2. The radiographs of calvaria at 12 weeks of post-transplantation. (a) The radiograph of the control on the right side and BCP group on the left side. The control group showed a slight radiopaque shadow in the radiograph. That of BCP group was almost reflected BCP particles in periphery of defects. (b) The radiograph of PRP loaded BCP group in bilateral defects. The radiodensity of PRP loaded BCP group was higher than that of BCP group. (c) The radiograph of BMP2/BCP group on the right and PRP/BMP2/BCP group on the left side. Periphery of the defect showed high radiodensity at BMP2/BCP group. PRP/BMP2/BCP group showed densified bone in the whole defect. (d) Quantitative evaluation of radiopaque areas at 6 and 12 weeks after transplantation. (Colors are visible in the online version of the article; http://dx.doi.org/10.3233/BME-130741.) 


\subsection{Histological evaluation}

The histology of the calvaria harvested at 12 weeks postoperatively is shown in Fig. 3. There was absolutely no bone formation in the control defects (Fig. 3(a)). The defects were filled with fibrous tissues. There were small amounts of new bone at the edge of the defects in the BCP group (Fig. 3(b)). Thin bone was observed on the periphery of the defects and the BCP particles were not present in the center portion of the defect. The histology of the PRP/BCP group showed a thin bone bridge at the bottom of each defect (Fig. 3(c)). The BCP particles remained throughout the defects. In contrast, the defects in the BMP2/BCP group were filled with abundant bone tissue that included the BCP particles (Fig. 3(d)). Moreover, in the PRP/BMP2/BCP group, prominent bone formations with BCP particles were clearly observed (Fig. 3(e)).

The results of the histological quantitative evaluation are illustrated in Fig. 4. Although the areas occupied by bone tissue showed no differences between the BCP and PRP/BCP groups at 6 weeks post-transplantation, the PRP/BCP group showed a significant increase in bone area compared with the BCP group at 12 weeks. Conversely, when BMP2 was employed, significant bone formation was shown

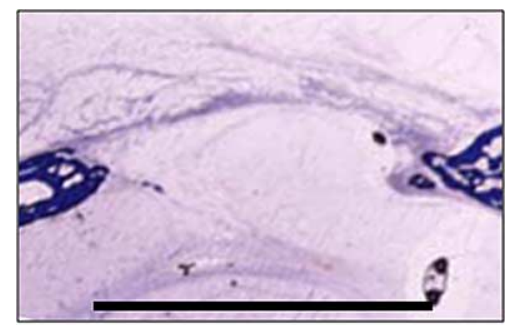

(a) Control (Empty)

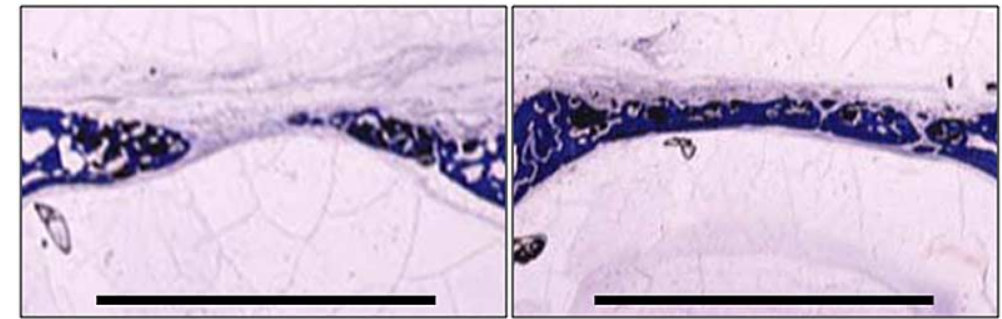

(b) $\mathrm{BCP}$

(c) $\mathrm{PRP} / \mathrm{BCP}$

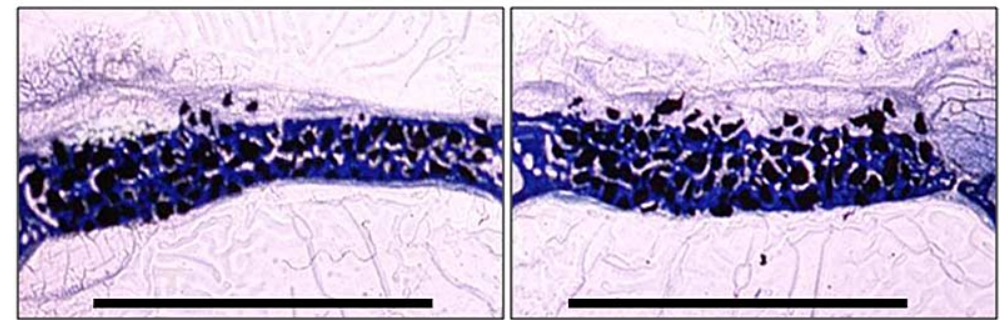

(d) $\mathrm{BMP2} / \mathrm{BCP}$

(e) $P R P / B M P 2 / B C P$

Fig. 3. The histological observation at 12 weeks of post-transplantation. (a) There were completely no bone formations in the control defects. (b) The histology of BCP group. There was thin bone in periphery of the defect. (c) The histology of PRP/BCP group. Bone bridge formation was seen at the bottom of defect. (d) The histology of BMP2/BCP group. The defect was filled with new bone with BCP particles. (e) The histology of PRP/BMP2/BCP group. Prominent bone formations with BCP particles were clearly observed. (Colors are visible in the online version of the article; http://dx.doi.org/10.3233/BME-130741.) 


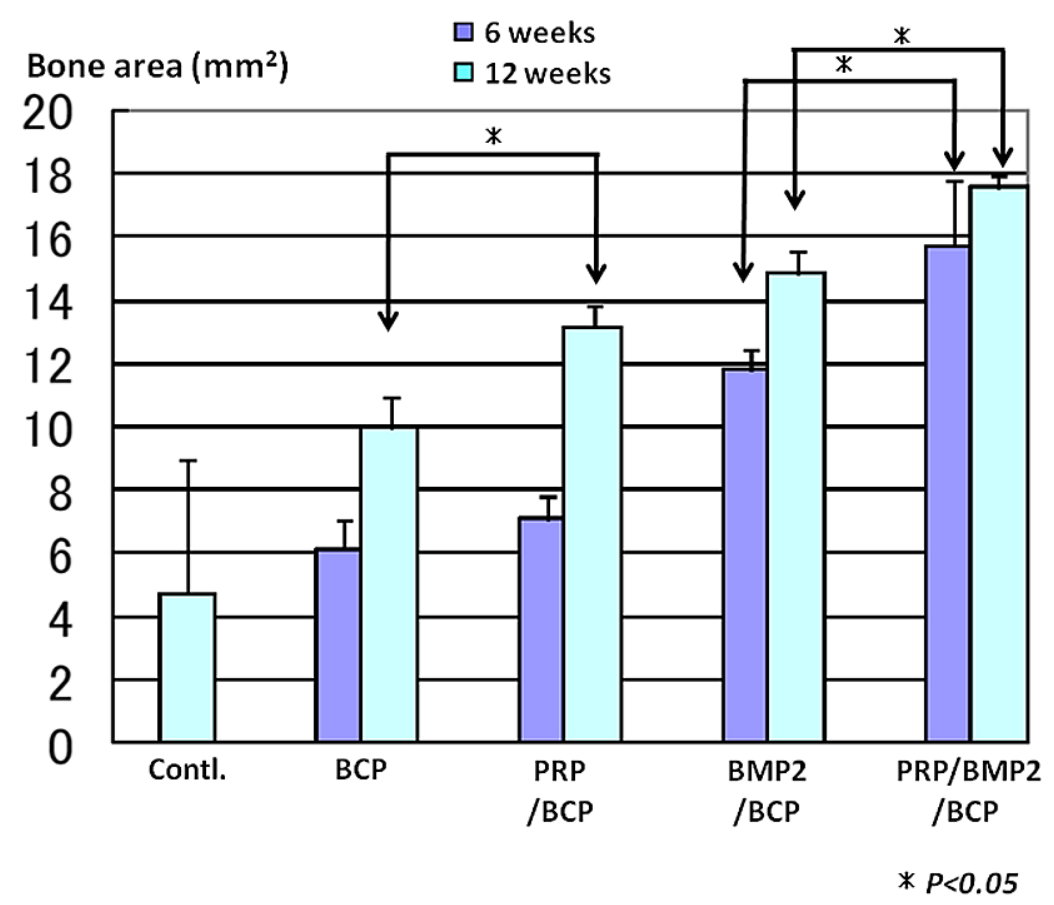

Fig. 4. The results of histological quantitative evaluation at 12 weeks of post-transplantation. PRP/BCP groups increased the bone area significantly compared with BCP groups at 12 weeks. When BMP2 was employed, significant bone formation was shown in BMP2/BCP groups. PRP promoted the bone formation further when combined with BMP2/BCP from 6 weeks of post-transplantation. (Colors are visible in the online version of the article; http://dx.doi.org/10.3233/BME-130741.)

in the BMP2/BCP group. However, PRP promoted the bone formation further when combined with BMP2/BCP from 6 weeks post-transplantation.

\section{Discussion}

The results from the present study show that PRP displays prominent osteoenhancive ability for rhBMP2-induced bone formation on an alloplastic substitute in rabbit calvaria. The successful treatment outcomes were: (1) conspicuous acceleration of bone formation from the early stage, (2) regeneration of abundant dense bone tissue and (3) demonstration of an unmistakable synergistic effect between PRP and rhBMP2 on BCP ceramics.

Recently, the development of bone substitutes based on osteogenic growth and differentiation factors with an optimal delivery system has drawn considerable attention $[9,10]$. Many synthetic biomaterials have been used for bone substitutes to date and optimal ones should have certain properties such as biocompatibility or osteoconductibility [10]. Regarding alloplastic bone substitutes, although they are known to lack osteoinductive properties, calcium phosphate ceramics, e.g., hydroxyapatite (HA) and tricalcium phosphate (TCP), have been used mostly because their chemical properties resemble those of bone matrix $[10,25]$. In this study, we used BCP ceramics that are composed of more soluble TCP and less soluble HA because a previous study reported their osteoinductive potentiality, which might be related to their degradation by macrophage phagocytosis [26]. However, BCP could induce a small amount of new bone only at the edge of the bone defects in this study. We must continue to focus on the 
development of ideal materials or methods that will add osteoinducibility to such alloplastic substitutes. Alloplastic bone substitutes have no cells in the graft and need more time for vascularization or cell population and matrix production compared with autogeneous bone grafts [27].

Regarding the acceleration of bone formation on BCP ceramics, both HA and TCP are well known to function as carriers of rhBMP2 [10,14,28,29]. Previous studies, as well as our own results, have demonstrated that the incorporation of rhBMP2 into these ceramics greatly accelerates the bone formation from the early stage until 4-6 weeks after transplantation $[10,28,30]$. Treatment of BCP ceramics with $10 \mu \mathrm{g}$ rhBMP2 induced bone formation two-fold higher compared with that of only BCP ceramics until 6 weeks. In contrast, PRP alone could not enhance any bone formation until 6 weeks in this study. Although the exact effect of PRP on osteogenesis is still controversial, several studies have shown that PRP has no positive effect on the early stages of bone healing in addition to the alloplastic or xenogenic substitutes [5,31,32]. The addition of PRP to the autogenous bone has a significant effect on bone regeneration from the initial stage by releasing several growth factors such as PDGF, TGF- $\beta$ and VEGF $[5,33,34]$. Growth factors contained in PRP stimulate angiogenesis and the proliferation of osteoprogenitor cells only in the very early period after transplantation [34,35]. Nevertheless, in this study, PRP could increase the bone area significantly at 12 weeks after transplantation compared with BCP grafts without PRP or rhBMP2. This result may be caused by the fibrin component of PRP that couples BCP particles and retains them better in local sites. As a histological observation, when only BCP ceramics were implanted, these particles were found only at the edge of the defects. However, the BCP particles remained throughout the regenerated bone bridge when PRP was applied to the BCP ceramics. However, PRP did not show the intended osteoinductive effect that rhBMP2 showed in the histological appearance.

Conversely, we found in this study that remarkable bone formation from an early stage was induced by supplying both PRP and rhBMP2. Indeed, the addition of these factors induced abundant bone tissue at 6 weeks after transplantation on the same level as the amount as rhBMP2-induced bone tissue at 12 weeks. Moreover, the radiological bone density at 6 weeks by combining PRP with rhBMP2 rose to the same level as that of rhBMP2-induced bone at 12 weeks. This result strongly suggests that rhBMP2 affected the increased osteoprogenitor cells by stimulation of growth factors contained in PRP. RhBMP2 may induce the osteoprogenitor cells to differentiate into fully functional mature osteoblasts [36-38]. Alternatively, the angiogenic effect of PRP may provide improved vascular perfusion for rhBMP2 to recruit more osteoprogenitor cells from the host bone marrow. A previous study demonstrated that the bone marrow-derived osteoprogenitor cells in circulating blood contribute to the ectopic bone formation induced by rhBMP2 [39]. Although PRP is known to contain some amount of BMP2 (approximately $0.03 \mathrm{ng} / \mathrm{ml}$ ) [40], such effects are resulted from increased amount of BMP by adding rhBMP2. Anyway, our data demonstrated the unmistakable synergistic effect produced by PRP and rhBMP2. The BCP ceramics could provide an appropriate environment for these two factors to generate their synergistic effect.

\section{Conclusions}

In conclusion, the present study revealed that PRP combined with BCP ceramics containing rhBMP2 can promote bone formation conspicuously in bony defects. Although the mechanisms that lead to the promotion of bone regeneration remain unclear, the enhancement of osteoinducibility in the alloplastic substitutes by the synergistic effect of PRP and rhBMP2 may provide a highly relevant approach to clinical bone reconstruction in the near future. We believe that PRP will support the function of a reduced 
concentration of rhBMP2. A lower dose of rhBMP2 will diminish the substantial swelling that may cause the obstruction of the airway as the result of direct implantation of high doses of rhBMP2 in oral and cervical areas.

\section{References}

[1] J.E. Hausamen and F.W. Neukam, Transplantation of bones, European Archives of oto-rhino-laryngology. Supplement 1 (1992), 163-165.

[2] J.T. Mellonig, Autogenous and allogeneic bone grafts in periodontal therapy, Critical Review in Oral Biology and Medicine 3 (1992), 333-352.

[3] S. Schultze-Mosgau, M. Keweloh, J. Wiltfang, P. Kessler and F.W. Neukam, Histomorphometric and densitometric changes in bone volume and structure after avascular bone grafting in the extremely atrophic maxilla, The British Journal of Oral \& Maxillofacial Surgery 39 (2001), 439-447.

[4] E. Nkenke, S. Schultze-Mosgau, M. Radespiel-Tröger, F. Kloss and F.W. Neukam, Morbidity of harvesting of chin grafts: a prospective study, Clinical Oral Implants Research 12 (2001), 495-502.

[5] J. Wiltfang, F.R. Kloss, P. Kessler, E. Nkenke, S. Schultze-Mosgau, R. Zimmermann and K.A. Schiegel, Effects of plateletrich plasma on bone healing in combination with autogenous bone and bone substitutes in critical-size defects, An animal experiment, Clinical Oral Implants Research 15 (2004), 187-193.

[6] J. Bateman, G. Intini, J. Margarone, S. Goodloe, P. Bush, S.E. Lynch and R. Dziak, Platelet-derived growth factor enhancement of two alloplastic bone matrices, Journal of Periodontology 76 (2005), 1833-1841.

[7] P.P. Skowronskim and Y.H. An, Bone graft materials in orthopaedics, Medical University of South Carolina Orthopaedic Journal 6 (2003), 58-66.

[8] E. Grageda, Platelet-rich plasma and bone graft materials: a review and a standardized research protocol, Implant Dentistry 13 (2004), 301-309.

[9] B.L. Eppley, W.S. Pietrzak and M.W. Blanton, Allograft and alloplastic bone substitutes: a review of science and technology for the craniomaxillofacial surgeon, The Journal of Craniofacial Surgery 16 (2005), 981-989.

[10] M.I. Alam, I. Asahina, K. Ohmamiuda, K. Takahashi, S. Yokota and S. Enomoto, Evaluation of ceramics composed of different hydroxyapatite to tricalcium phosphate ratios as carriers for rhBMP-2, Biomaterials 22 (2001), 1643-1651.

[11] H.C. Lim, J.Y. Sohn, J.C. Park, Y.J. Um, U.W. Jung, C.S. Kim, Y.K. Lee and S.H. Choi, Osteoconductive effects of calcium phosphate glass cement grafts in rabbit calvarial defects, Journal of Biomedical Materials Research Part B, Applied Biomaterials 95 (2010), 47-52.

[12] A.H. Reddi, Initiation of fracture repair by bone morphogenetic proteins, Clinical Orthopaedics and Related Research 355(Suppl) (1998), S66-S72.

[13] R.E. Jung, R. Glauser, P. Schärer, C.H. Hämmerle, H.F. Sailer and F.E. Weber, Effect of rhBMP-2 on guided bone regeneration in humans, Clinical Oral Implants Research 14 (2003), 556-568.

[14] R.E. Jung, F.E. Weber, D.S. Thoma, M. Ehrbar, D.L. Cochran and C.H. Hämmerle, Bone morphogenetic protein-2 enhances bone formation when delivered by a synthetic matrix containing hydroxyapatite/tricalciumphosphate, Clinical Oral Implants Research 19 (2008), 188-195.

[15] F. Schwarz, D. Ferrari, M. Sager, M. Herten, B. Hartig and J. Becker, Guided bone regeneration using rhGDF-5- and rhBMP-2-coated natural bone mineral in rat calvarial defects, Clinical Oral Implants Research 20 (2009), 1219-1230.

[16] H. Ikeda, Y. Sumita, M. Ikeda, H. Ikeda, T. Okumura, E. Sakai, M. Nishimura and I. Asahina, Engineering bone formation from human dental pulp- and periodontal ligament-derived cells, Annals of Biomedical Engineering 39 (2011), 26-34.

[17] D.M. Toriumi, H.S. Kotler, D.P. Luxenberg, M.E. Holtrop and E.A. Wang, Mandibular reconstruction with a recombinant bone-inducing factor. Functional, histologic, and biomechanical evaluation, Archives Otolaryngology - Head \& Neck Surgery 117 (1991), 1101-1112.

[18] P.J. Boyne, R. Nath and A. Nakamura, Human recombinant BMP-2 in osseous reconstruction of simulated cleft palate defects, The British Journal of Oral \& Maxillofacial Surgery 36 (1998), 84-90.

[19] P.J. Boyne, A. Nakamura and S. Shabahang, Evaluation of the long-term effect of function on rhBMP-2 regenerated hemimandibulectomy defects, The British Journal of Oral \& Maxillofacial Surgery 37 (1999), 344-352.

[20] V.V. Patel, L. Zhao, P. Wong, B.B. Pradhan, H.W. Bae, L. Kanim and R.B. Delamarter, An in vitro and in vivo analysis of fibrin glue use to control bone morphogenetic protein diffusion and bone morphogenetic protein-stimulated bone growth, The Spine Journal 6 (2006), 397-403.

[21] K.L. Yaremchuk, M.S. Toma, M.L. Somers and E, Peterson, Acute airway obstruction in cervical spinal procedures with bone morphogenetic proteins, The Laryngoscope 120 (2010), 1954-1957. 
[22] A. Tomoyasu, K. Higashio, K. Kanomata, M. Goto, K. Kodaira, H. Serizawa, T. Suda, A. Nakamura, J. Nojima, T. Fukuda and T. Katagiri, Platelet-rich plasma stimulates osteoblastic differentiation in the presence of BMPs, Biochemical Biophysical Research Communications 361 (2007), 62-67.

[23] T.F. Tözüm and B. Demiralp, Platelet-rich plasma: a promising innovation in dentistry, Journal Canadian Dental Association 69 (2003), 664.

[24] L. Boyapati and H.L. Wang, The role of platelet-rich plasma in sinus augmentation: a critical review, Implant Dentistry 15 (2006), 160-170.

[25] A. El-Ghannam, Bone reconstruction: from bioceramics to tissue engineering, Expert Review of Medical Devices 2 (2005), 87-101.

[26] B.H. Fellah, B. Delorme, J. Sohier, D. Magne, P. Hardouin and P. Layrolle, Macrophage and osteoblast responses to biphasic calcium phosphate microparticles, Journal of Biomedical Materials Research Part A 93 (2010), 1588-1595.

[27] E.S. Kim, J.J. Kim and E.J. Park, Angiogenic factor-enriched platelet-rich plasma enhances in vivo bone formation around alloplastic graft material, The Journal of Advanced Prosthodontics 2 (2010), 7-13.

[28] I. Ono, H. Gunji, F. Kaneko, T. Saito and Y. Kuboki, Efficacy of hydroxyapatite ceramic as a carrier for recombinant human bone morphogenetic protein, The Journal of Craniofacial Surgery 6 (1995), 238-244.

[29] J. Tazaki, M. Murata, T. Akazawa, M. Yamamoto, K. Ito, M. Arisue, T. Shibata and Y. Tabata, BMP-2 release and doseresponse studies in hydroxyapatite and beta-tricalcium phosphate, Bio-Medical Materials and Engineering 19 (2009), 141-146.

[30] Y.M. Lee, S.H. Nam, Y.J. Seol, T.I. Kim, S.J. Lee, Y. Ku, I.C. Rhyu, C.P. Chung, S.B. Han and S.M. Choi, Enhanced bone augmentation by controlled release of recombinant human bone morphogenetic protein-2 from bioabsorbable membranes, Journal of Periodontology 74 (2003), 865-872.

[31] A.S. Plachokova, J. van den Dolder, P.J. Stoelinga and J.A. Jansen, The bone regenerative effect of platelet-rich plasma in combination with an osteoconductive material in rat cranial defects, Clinical Oral Implants Research 17 (2006), 305-311.

[32] A.S. Plachokova, J. van den Dolder, P.J. Stoelinga and J.A. Jansen, Early effect of platelet-rich plasma on bone healing in combination with an osteoconductive material in rat cranial defects. Clinical Oral Implants Research 18 (2007), $244-251$.

[33] R.E. Marx, E.R. Carlson, R.M. Eichstaedt, S.R. Schimmele, J.E. Strauss and K.R. Gergeff, Platelet-rich plasma: Growth factor enhancement for bone grafts, Oral Surgery, Oral Medicine, Oral Pathology, Oral Radiology, and Endodontics $\mathbf{8 5}$ (1998), 638-646.

[34] R.E. Jung, H.G. Schmoekel, R. Zwahlen, V. Kokovic, C.H. Hammerle and F.E. Weber, Platelet-rich plasma and fibrin as delivery systems for recombinant human bone morphogenetic protein-2, Clinical Oral Implants Research 16 (2005), 676-682.

[35] R.E. Marx, Platelet-rich plasma (PRP): what is PRP and what is not PRP?, Implant Dentistry 10 (2001), 225-228.

[36] Y. Hiraki, H. Inoue, C. Shigeno, Y. Sanma, H. Bentz, D.M. Rosen, A. Asada and F. Suzuki, Bone morphogenetic proteins (BMP-2 and BMP-3) promote growth and expression of the differentiated phenotype of rabbit chondrocytes and osteoblastic MC3T3-E1 cells in vitro, Journal of Bone and Mineral Research 6 (1991), 1373-1385.

[37] Y. Takuwa, C. Ohse, E.A. Wang, J.M. Wozney and K. Yamashita, Bone morphogenetic protein-2 stimulates alkaline phosphatase activity and collagen synthesis in cultured osteoblastic cells, MC3T3-E1, Biochemical Biophysical Research Communications 174 (1991), 96-101.

[38] F.J. Hughes, J. Collyer, M. Stanfield and S.A. Goodman, The effects of bone morphogenetic protein-2, -4 , and -6 on differentiation of rat osteoblast cells in vitro, Endocrinology 136 (1995), 2671-2677.

[39] S. Otsuru, K. Tamai, T. Yamazaki, H. Yoshikawa and Y. Kaneda, Circulating bone marrow-derived osteoblast progenitor cells are recruited to the bone-forming site by the CXCR4/stromal cell-derived factor-1 pathway, Stem Cells 26 (2008), 223-234.

[40] G. Schmidmaier, S. Herrmann, J. Green, T. Weber, A. Scharfenberger, N.P. Haas and B. Wildemann, Quantitative assessment of growth factors in reaming aspirate, iliac crest, and platelet preparation, Bone 39 (2006), 1156-1163. 